\title{
Potent Intracellular Knock Down of Hepatitis B Virus X RNA by Catalytic Hammerhead Ribozymes or DNA-Enzymes with Antisense DNA-Oligo- nucleotides or 10-23 DNA-Enzymes that Powerfully Augment In Vitro Sequence-Specific Cleavage Activities
}

\author{
Nidhi Gupta, Aalia S. Bano, Yogeshwar Sharma, Vikas Sood and Akhil C. Banerjea* \\ National Institute of Immunology, Department of Virology, New Delhi-110067, India
}

\begin{abstract}
Novel antiviral approaches are needed to control Hepatitis B virus infection worldwide. X protein of this virus activates various promoters and is strongly associated with hepatocellular carcinoma. Although several groups, including ours, reported sequence-specific cleavage of X RNA by either ribozymes (Rzs) or DNA-enzymes (Dzs) earlier, but none of these studies reported $100 \%$ in vitro cleavage of the full-length X RNA. We reasoned that by melting the secondary structures near the Rz/Dz cleavage site with specific antisense DNA oligonucleotides (ODNs) or 10-23 Dz, it may be possible to achieve this objective. Hammerhead motif containing Rz-170 specific for X RNA was constructed by recombinant techniques and Dz-237 was synthesized using the 10-23 catalytic motif. When specific ODNs or 10-23 Dzs were included in the cleavage reaction with either Rz-170 or Dz-237, increased cleavage was observed in a dose-dependent manner which often resulted in almost complete in vitro cleavage of the target RNA. Rz-170 in combination with specific ODNs caused potent intracellular reduction of HBx RNA. Thus, the cleavage activity of catalytic nucleic acids (Rzs or Dzs) can be increased significantly by specific ODNs or Dzs and this treatment also results in potent intracellular target RNA reduction. These findings have important therapeutic implications.
\end{abstract}

\section{INTRODUCTION}

Ribozymes (Rzs) are short catalytic RNA molecules that possess the ability to cleave the target RNA in a sequencespecific manner and control splicing reactions (Cech, 1987) $[1,2,3,4]$. Although several types of catalytic motifs have been described, the hammerhead $\mathrm{Rz}$ being the smallest and with minimal target sequence requirement, has been exploited extensively for variety of purposes including therapeutic applications $[5,6,7,8]$. On the other hand, Deoxyribozymes or DNA-enzymes (Dzs), as originally [9] are short DNA molecules that can be designed to cleave any target RNA in a sequence-specific and catalytic manner $[10,11,12]$. Over the years several kinds of Dzs with unique catalytic motifs have been described but Dz possessing the 10-23 catalytic motif has been exploited extensively by several investigators [11]. This Dz possesses a conserved 15 nucleotide long 10-23 catalytic motif and is able to cleave any target RNA between purine and pyrimidine under in vitro and in vivo conditions. Their ability to cleave any target RNA has been exploited for knocking down gene expression against variety of target genes including HIV-1 genes $[11,13,14]$ with varying outcomes. In some instances very efficient intracellular gene inhibition was observed with 10 $23 \mathrm{Dz}$ [15]. We also reported earlier that $10-23 \mathrm{Dz}$ was more effective in cleaving the full-length CCR5 (HIV-1 coreceptor) RNA compared to a hammerhead ribozyme $[16,17]$ carried out a comparative study between hammerhead ribozymes (Rzs) and Dzs that were targeted against the same sequences and concluded that some sequences were cleaved

*Address correspondence to this author at the National Institute of Immunology, Department of Virology, New Delhi-110067, India;

E-mail: akhil@nii.res.in better by Rzs and some by Dzs. There are several other nucleic-acid based approaches that have been used for specific inhibition of target genes. The catalytic ribozymes, aptamers, antisense DNA or RNA or small interfering RNAs (siRNAs) have been used for inhibiting the expression of foreign genes including HIV-1 replication [8]. Physiologically relevant RNAs are usually long and consist of multiple stem-loop structures and up to $90 \%$ putatitive cleavage sites were earlier shown to be in inaccessible to either Rz or Dz mediated cleavage [18]. Earlier, unwinding activity of an RNA helicase was used to increase the cleavage potential of Rzs [19]. Collectively, all these studies suggest that not all the target sites are available for cleavage by a single kind of catalytic nucleic-acid molecule most probably because the secondary and tertiary structures in the target RNA prevent optimal Watson-Crick base pairing with Rzs or Dzs. Introduction of locked nucleic-acids in antisense design resulted in enhancing its stability [20] and when incorporated in the substrate recognition arms of a Dz, it improved the catalytic efficiency [21].

Earlier few nucleic-acid based approaches were used to modulate the cleavage activity of the Dzs and Rzs with moderate success but none of them were tested for bio-efficacy. Oligonucleotide effectors (regulators) that bind to both enzyme and substrate were used to regulate the catalytic activity of Rzs \& Dzs [22, 23]. Whether oligodeoxynucleotides (ODNs) that hybridize specifically to the target RNA alone could modulate in vitro and in vivo the catalytic activity of Rzs or Dzs have not been earlier attempted.[24] reported enhancement of ribozyme catalytic activity by a contiguous oligonucleotide (facilitator) and by 2'-O-methylation. Oligonucleotide facilitators have been used to either enhance 
[25] or even inhibit catalytic activity of Rzs [26]. We recently reported $\mathrm{Dz}$ mediated augmentation of in vitro cleavage of a truncated HIV-1 Gag gene by specific antisense molecules [27]. Oligonucleotide facilitators have earlier been used to enhance hammerhead ribozyme mediated cleavage of long RNA substrates with multiple-turnover activity [28]. Although all the above approaches resulted in enhanced cleavage of the target RNA under in vitro conditions, it was not known if combinations of ribozymes or DNA-enzymes with facilitators could be exploited to down regulate intracellular levels of the target RNAs.

In the present study, we have identified and characterized those ODNs and Dzs that significantly enhanced the Rz and Dz mediated cleavage of full-length X RNA (HBx RNA) of hepatitis $\mathrm{B}$ virus (HBV) on one hand and inhibit intracellular expression of the target RNA in a liver specific cell line, HepG2 on the other. The Hepatitis B virus X protein (HBx) acts as a powerful transactivator for several genes including HBV genes and is known to be strongly associated with hepatocellular carcinoma $[29,30]$. These results are potentially important for therapeutic purposes for the treatment of HBV infections against which there are no effective antiviral treatment available.

\section{METHODOLOGY}

\section{Cloning of HBx Gene and In Vitro Transcription}

Plasmid pSG5.HBx encoding the $\mathrm{X}$ gene of HBV [31] was a kind gift from Vijay Kumar, ICGEB, New Delhi, India. The entire gene is placed under SV40 and T7 promoters (Fig. 1). The former promoter is used for intracellular expression and the latter was used for obtaining in vitro transcripts using the transcription kit from Promega Biotech., as described earlier [32]. After linearization with appropriate enzyme at the 3'-end, full-length X RNA will be synthesized.

\section{Construction and Cloning of HBx-Rz-170 Targeted Against X Gene}

The construction of Rz-170 (Fig. 2A) has been described by us in detail previously [33]. It possessed the hammerhead motif and targeted against the GUC sequence. EcoR 1 and $B a m H 1$ restriction sites were engineered at the ends of the hybridizing arms of the $\mathrm{Rz}$ which facilitated cloning it into pcDNA3 expression vector (Promega). This placed the $\mathrm{Rz}$ under $\mathrm{T} 7$ and $\mathrm{CMV}$ promoter.

\section{In Vitro Cleavage of Target RNA with $\mathrm{Rz}$}

In vitro transcription of the linearized plasmid DNA was carried out in the presence of labeled UTP using T7 RNA polymerase. The cleavage reaction was initiated by adding equimolar amounts (100 pmoles each) of the labeled target RNA and unlabeled $\mathrm{Rz}$ in a reaction buffer containing $50 \mathrm{mM}$ Tris $/ \mathrm{HCl}, \mathrm{pH} 7.5$, in a volume of $10 \mu 1$. The reaction mixture was heated briefly at $94^{\circ} \mathrm{C}$ and the cleavage reaction was initiated by adding $\mathrm{MgCl}_{2}$ (final concentration $10 \mathrm{mM}$ ) at $37^{\circ} \mathrm{C}$ for 2 hours. The cleaved RNA fragments were subjected to gel analysis as described before [34]. The radioactive RNA bands on the X-Ray film were quantitated by densitometry (GS-710 Calibrated Imaging Densitometer, BioRad, Hercules, CA, USA).

\section{Primers \& DNA-Enzyme}

All the 20nt long antisense oligonucleotides (ODNs) were synthesized chemically and obtained from Sigma Genosys (The Woodlands, TX). The conserved 15 nt long (5'-GGCTAGCTACAACGA) 10-23 catalytic motif was flanked on both sides by substrate-binding arms of the $\mathrm{Dz}$ that were made complementary to the target RNA. We have earlier described in detail the construction of Dz-237 that cleaved the X RNA specifically into two fragments (Fig. 2B) [35]. Dz-155, wild-type and mutant versions of Dz-192 were also assembled using 10-23 catalytic motif that possessed a single nucleotide substitution ( $\mathrm{G}$ to $\mathrm{C}$ ) in the $10-23$ catalytic motif (Fig. 3B). This change is known to render the Dz catalytically inactive [35].

\section{Dz-Mediated Cleavage}

Equivalent amounts of unlabeled $\mathrm{Dz}$ and labeled substrate RNA (100 pmoles each) were allowed to interact in a final volume of $10 \mu \mathrm{l}$ in a buffer containing $50 \mathrm{mM}$ Tris. $\mathrm{HCl}$, $\mathrm{pH} 7.5$ in presence of $10 \mathrm{mM} \mathrm{MgCl}_{2}$ (standard conditions) as described earlier [9] for 2 hours at $37^{\circ} \mathrm{C}$ in the absence or in the presence of indicated amounts of ODNs. The cleaved products were resolved by gel analysis and cleavage efficiency was determined as described earlier [34, 35].
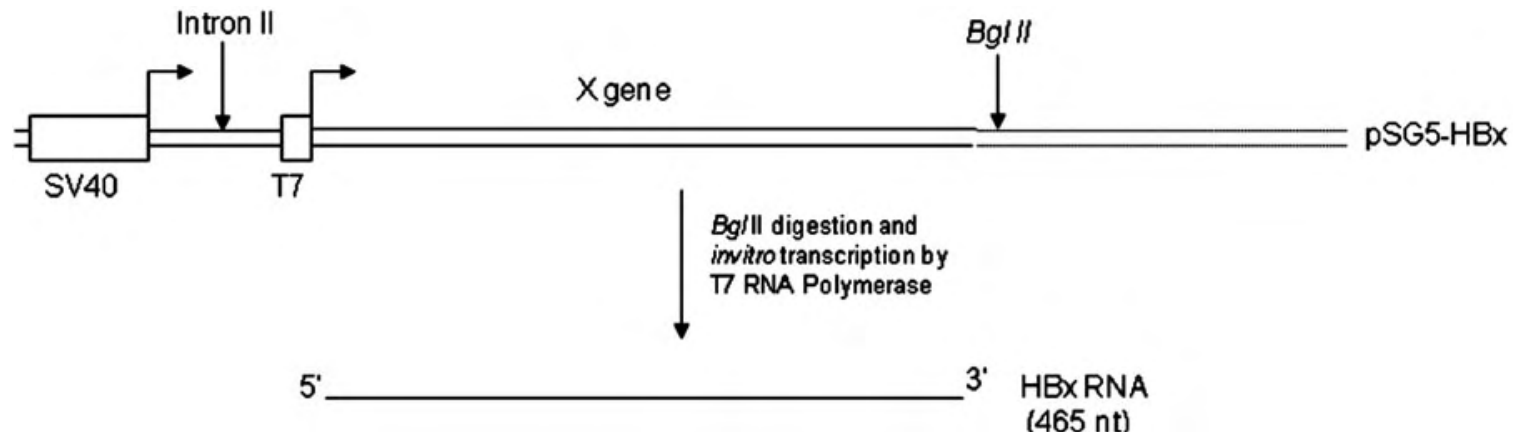

(465 nt)

Fig. (1). Full-length HBx encoding DNA and in vitro transcription.

Plasmid pSG5.HBx (Kumar et al., 1996) contains the full length X gene of hepatitis B virus. It is placed downstream of the powerful SV40 and T7 promoter of the expression vector pSG5 (Clonetech, Palo, Alto, CA, USA). After linearizing it with $B g l$ II restriction enzyme, a 465nt long transcript is generated by in vitro transcription using T7 RNA polymerase. 


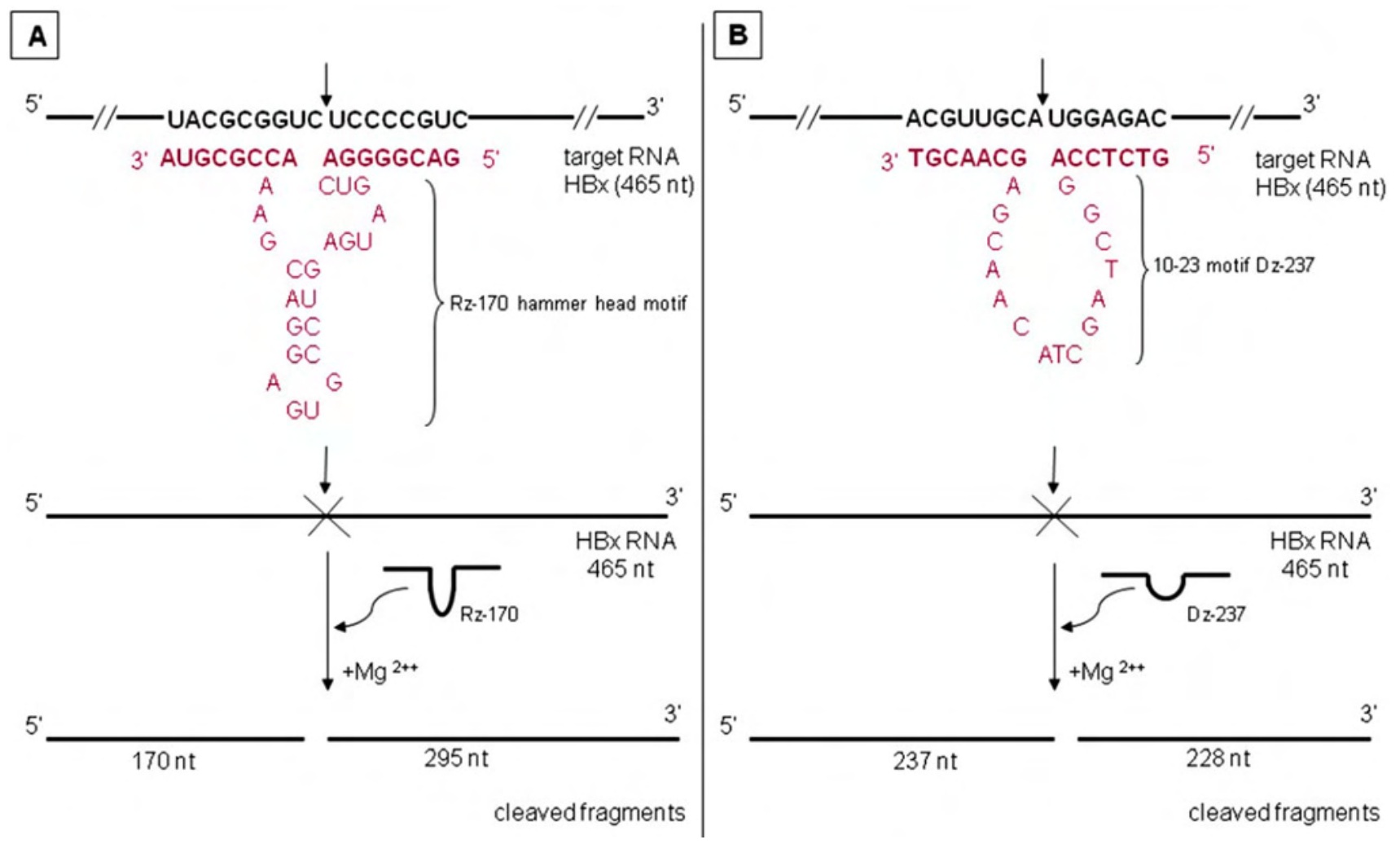

Fig. (2). Sequence of the target RNA against which Rz-170 and Dz-237 were designed are shown along with their specific cleavage products.

For constructing Rz-170, eight bases long hybridizing arms are made complementary to the target RNA to provide specificity (Panel A). The hammerhead catalytic motif is same as described before (Shahi et al., 2001). The site of cleavage in the target RNA is shown by an arrow. When the full length HBx RNA is subjected to cleavage by Rz-170 in presence of $\mathrm{MgCl}_{2}$, two specific RNA fragments are expected that are170 and 295nt long. Panel B: 10-23 catalytic motif containing Dz-237 was chemically synthesized that targeted AU dinucleotide in the target RNA for cleavage. 7 bases long hybridizing arms were made complementary to the target RNA to provide specificity. The expected cleavage products generated due to the action of $\mathrm{Dz}$ are shown.

\section{Intracellular Inhibition of the Expression of Target RNA by $\mathrm{Rz}+$ ODNs}

The intracellular decrease in $\mathrm{HBx}$-specific RNA after cotransfection into HepG2 cells with substrate encoding DNA (pSG5.HBx) + Rz in the presence or absence of ODNs were monitored by RT-PCR based assays. To ensure uniform transfection efficiency, we always transfected equal amounts of reporter gene containing plasmid (pSV- $\beta$-gal, Promega). We repeatedly obtained about $70-80 \%$ transfection efficiency. All the plasmid DNAs used was Qiagen column purified which showed no toxicity under our experimental conditions. Several dilutions were initially made to determine the linear range for PCR-amplified products. Total RNA was isolated using TRIZOL reagent (GIBCO-BRL) following the procedure described by the manufacturer and divided into two equal sets. One set was used for estimating the levels of full-length X RNA using HBx1 and HBx2 primers and the second set was used for estimating the levels of the house keeping gene, human glyceraldehyde-phosphodehydrogenase (HuGAPDH) as described earlier [36]. The following primers were used for estimating the intracellular levels of full-length X RNA.

(1) Forward (HBx1): 5'-TTAGGCAGAGGTGAAAAAGTT GCATGGTGCTGG .

(2) Reverse(HBx2):5' - TGGCTGCTAGGCTGTACTGCCA
ACTGGATCCTTCG

\section{Western Blot Analysis}

$1 \times 10^{6}$ HepG2 cells were grown to $80 \%$ confluency in a six well plate. They were transfected with either Rz-170 $(1 \mu \mathrm{g} / \mathrm{ml})$ alone or in the presence of increasing concentrations of ODN \#1 and \# 2 in a final volume of $1 \mathrm{ml}$ for 48 hours along with $1 \mu \mathrm{g}$ of $\mathrm{X}$ gene encoding DNA, pSG5.HBx, using lipofectin (Invitrogen). Cell lysates were prepared and equivalent amounts of proteins were loaded in each lane as described earlier [35, 33]. They were subjected to gel analysis and transferred on to a nitrocellulose membrane. Rabbit polyclonal antibody to HBx (Biovendor, NJ, USA) was used as a primary antibody in 1:500 dilution in PBS pH 7.2 containing $0.1 \%$ Tween 20 . For $\beta$-actin as control, mouse raised monoclonal antibody (Calbiochem, CA ,USA) was used as primary with 1:10,000 dilution in PBS-T. The amounts of pSG5.HBx DNA was kept constant in each experimental lane at $1 \mu \mathrm{g} / \mathrm{ml}$. The absolute amounts of DNA introduced in each well was made equivalent by adding unrelated DNAs.

\section{Inhibition of X Protein Mediated HIV-1 LTR Activation with Rz and ODNs or Dzs}

HEK 293 or HepG2 cells were cotransfected with $100 \mathrm{ng}$ of pBS-LTR-B-Luciferase (henceforth referred to as pLTR$B$ - the Luciferase reporter gene is placed down stream of the HIV-1 LTR-B promoter) (obtained from AIDS Research and Reference Reagent Program of NIH, MD, USA) plasmid 

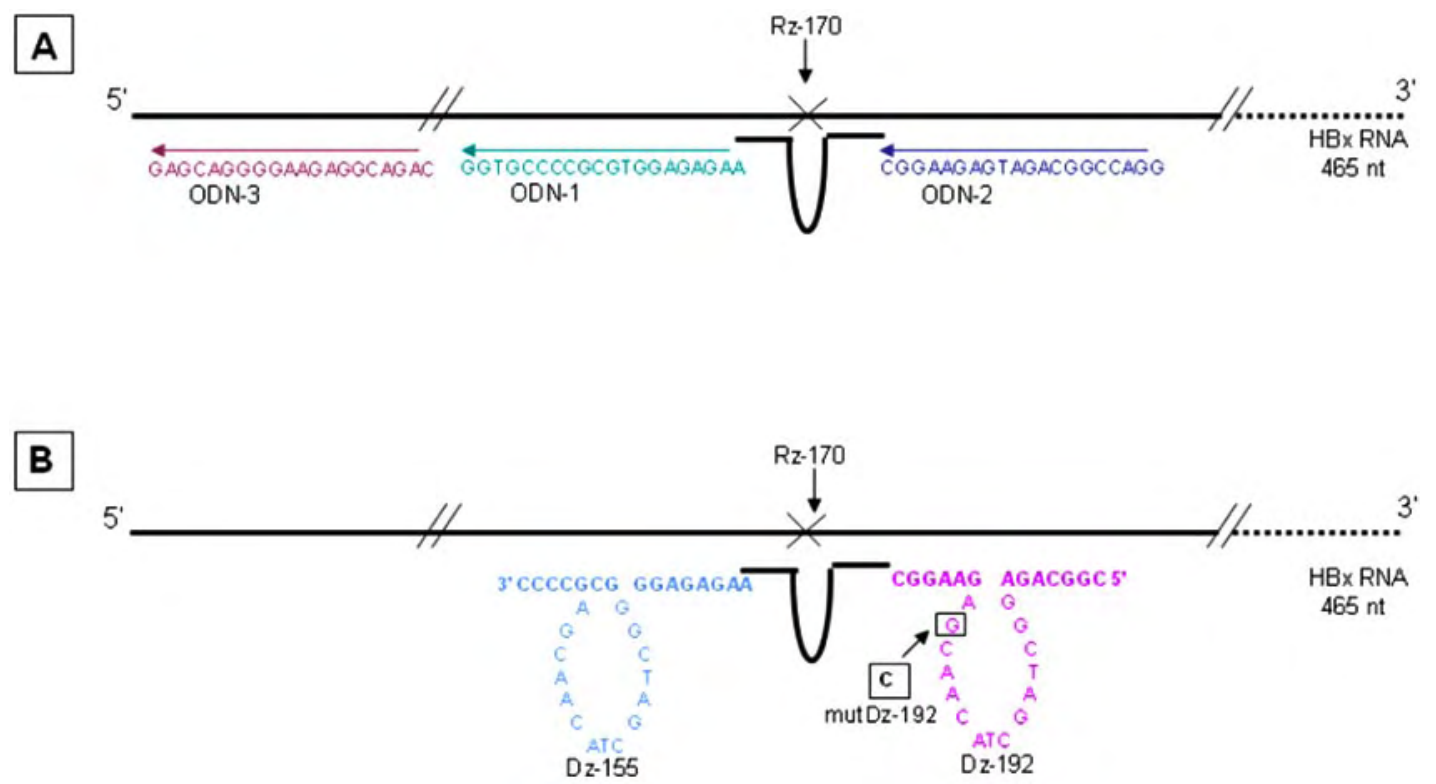

Fig. (3). ODNs and Dzs designed to augment Rz-170 mediated cleavage products.

Panel A: Three 20 nucleotides long antisense oligodeoxynucleotides (ODNs) (\#1 to \# 3) were synthesized. Two of them (ODN \#1 and 2) were targeted to hybridize immediately next to the hybridizing arms of the Rz-170 and their sequences are shown. ODN \#3 was 33nt away from the Rz cleavage site and its sequence is also shown. All the oligonucleotides were chemically synthesized and obtained from Sigma Genosys. Panel B: Two Dzs (Dz-155 and 192) possessing 10-23 catalytic motif, were synthesized immediately upstream and downstream of Rz-170. A mutant (disabled) version of Dz-192 was synthesized by substituting a C residue in place of G in the catalytic motif.

in the presence of indicated amounts of ODNs or Dzs in wells that received 10 ọng of pSG5.HBx plasmid DNA that was kept constant in all the wells. Lipofectin was used to introduce the mixture of various DNA combinations and cell lysates were prepared 24 hours later using $1 x$ reporter lysis buffer (Promega). The extent of Luciferase activity was determined according to the manufacturer's instructions (Promega). A control reporter plasmid (pSV- $\beta$-gal, Promega) was always included to ensure uniform transfection efficiency.

\section{RESULTS}

\section{In Vitro Synthesis of Full-Length HBx RNA by T7 RNA Polymerase}

Plasmid pSG5-HBx (Fig. 1) was linearized with $B g l$ II restriction enzyme and when subjected to in vitro transcription using T7 RNA polymerase, a 465nt long full-length $\mathrm{HBx}$ transcript is expected using the Riboprobe transcription kit (Promega Biotech., WI, USA) in the presence of ${ }^{32} \mathrm{P}$ UTP. Transcripts were subjected to gel analysis and dried gels were exposed to X Ray.

\section{Rz-170 Mediated Cleavage of HBx RNA in the Presence of Upstream and Downstream ODNs}

Entire sequence of the Rz-170 along with the target RNA is shown in Fig. (2A). The target sequence was GUC located at nucleotide position 170 in the $\mathrm{HBx}$ gene. The 22nt long hammerhead motif was flanked with eight nucleotides long hybridizing arms on either side of the target sequence that were made complementary to the target RNA to provide specificity. When an equimolar amount (100pmoles each) of Rz-170 is used for cleaving a full-length HBx RNA (465nt), specific RNA fragments (170 and 295 bases long) are expected (Fig. 2A). In order to increase the Rz-170 mediated cleaved products, 3 antisense ODNs, each 20nt long, were synthesized. Two of these ODNs (\#1 \& \#2) were designed to hybridize immediately adjacent to the two hybridizing arms of the hammerhead Rz-170. We designed another ODN (ODN\#3) that was 33 nt upstream from the Rz-170 cleavage site (Fig. 3, panel A). In the similar manner, 10-23 catalytic motif containing Dzs were synthesized immediately upstream and downstream of the Rz-170. Exact sequence of these Dzs along with the mutant (disabled Dz-192) is shown in Fig. (3, panel B). Rz-170 mediated cleavage reaction was performed in the presence of indicated amount of ODNs individually and the results are shown in Fig. (4A). Lane 1 shows the synthesis of uniformly labeled full-length (465nt long) HBx RNA. When equimolar amounts (100 pmoles each) of $\mathrm{Rz}$ and labeled substrate RNA were used for cleavage under standard conditions [7, 12, 31], two specifically cleaved fragments (295 and 170nt long RNA fragments) were obtained (lane 2). When the same cleavage reaction was carried out in the presence of 10pmoles (lane 3); 50pmoles (lane 4); and 100pmoles (lane5) of ODN\#1, a dose-dependent decrease in the input substrate RNA was observed. Approximately 4-fold decrease was observed in the input substrate RNA when lane 5 was compared with lane 2, and 2.5 fold reduction when lanes 3 and 4 were compared with lane2 (Fig. 4A). In the similar manner, the ODN\#2 also caused a dose-dependent decrease in the substrate RNA when similar amounts were used (10pmoles lane 6; 50pmoles - lane 7; 100pmoles - lane 8). Note almost complete disappearance of the input substrate RNA in lane 8. We conclude that ODNs that hybridized immediately upstream and downstream of Rz-170, were able to significantly 
A.

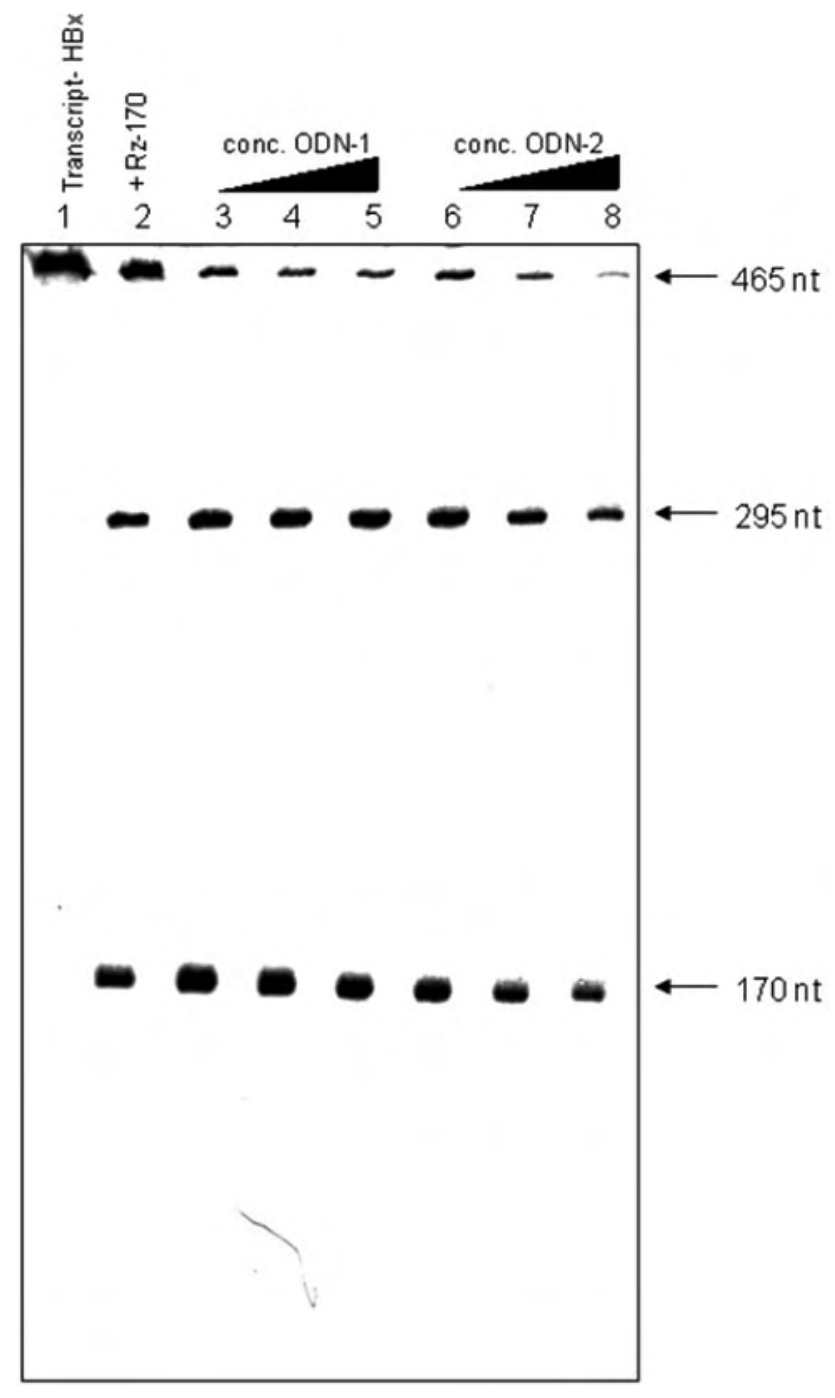

B.

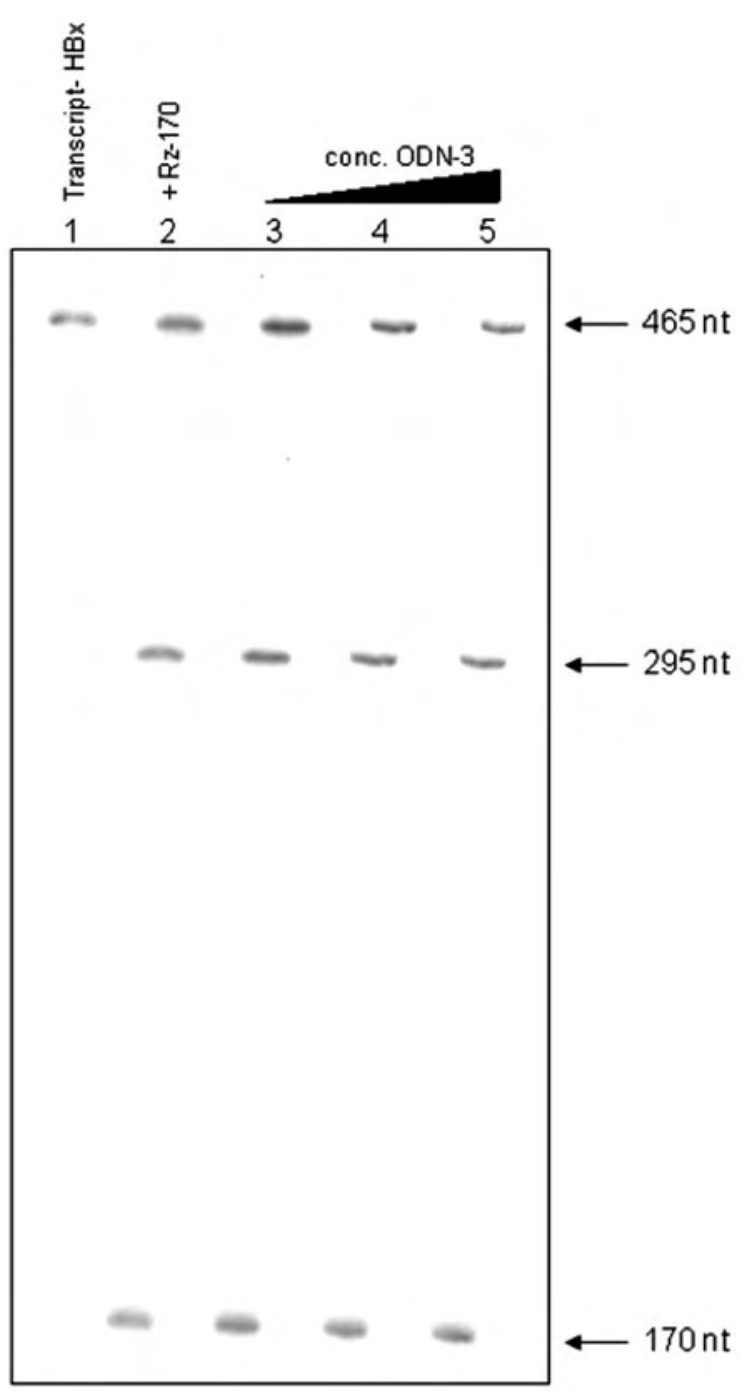

Fig. (4A). Augmentation of Rz-170 mediated cleavage by specific ODNs.

ane 1 shows the synthesis of uniformly labeled 465nt long HBx RNA. When equimolar amounts (100pmoles each) of substrate and Rz-170 were mixed in presence of $10 \mathrm{mM} \mathrm{MgCl}_{2}$, specific cleavage products (295 and 170nt long RNA fragments) were observed (lane 2). When the same cleavage reactions were carried out in presence of increased amounts of ODN \#1 (10 pmoles - lane 3; 50 pmoles -lane 4; 100 pmoles lane 5) or ODN \#2 (10 pmoles -lane 6; 50 pmoles -lane 7 and 100 pmoles -lane 8), a significant reduction in the input substrate RNA was observed with concomitant increase in the cleavage products.

Fig. (4B). ODN\#3 fails to augment Rz-170 mediated cleavage products.

When the same substrate RNA (lane 1) was subjected to Rz-170 mediated cleavage in the presence of increasing amounts of ODN \#3 (lane 3 -1pmole; lane 4 -10pmoles; lane 5-100pmoles and lane 6 -200pmoles), no significant increase in the cleavage products were observed when compared with Rz-170 mediated cleavage only (lane 2).

augment the Rz-170 mediated cleavage and that ODN\#2 was more effective than ODN\#1.

\section{Antisense ODN \#3 Targeted to Hybridize 33nt Away from the Rz-170 Cleavage Site Fails to Augment its Cleavage}

The nature of the experiment is same as described in Fig. (4A). Briefly, Rz-170 mediated cleavage of the target RNA (Fig. 4B, lane 1) was studied in the presence of increasing amounts of ODN\#3 (Fig. 3A) and the results are shown in Fig. (4B). Lane 2 exhibits the cleavage obtained with equimolar amounts (100pmoles) of labeled substrate and Rz-170. When increasing amounts of ODN\#3 (1pmole - lane 3; 10pmole - lane 4; 100pmoles - lane 5 and 200pmoles - lane 6) were included in the cleavage reaction, the extent of cleavage remained unchanged. We conclude that ODN that hybridized $33 \mathrm{nt}$ upstream from the Rz-170 cleavage site failed to augment Rz-170 mediated cleavage. 


\section{Only Downstream Dz (Dz-192) Significantly Enhanced Rz-170 Mediated Cleavage}

Since we observed significant enhancement of Rz-170 mediated cleavage products by the two ODNs (\#1 \& \#2) that hybridized immediately upstream and downstream to the hybridizing arms of the Rz, we wanted to find out if the two Dzs (Dz-155 and Dz-192) that were designed in a similar manner (Fig. 3B) could also enhance the Rz-170 mediated cleavage. The various cleavage products (including partially cleaved products) that are expected due to the combined actions of $\mathrm{Rz}+\mathrm{Dz}-155$ and $\mathrm{Rz}+\mathrm{Dz}-192$ are shown schematically in Fig. (5). The reaction conditions were same as described for Fig. (4A) and the results are shown in Fig. (6A). Lane 1 shows the labeled full-length in vitro synthesized $\mathrm{HBx}$ RNA as described earlier. Lane 2 shows sequencespecific cleavage of the target RNA when equimolar amounts of $\mathrm{Rz}$ were used. In presence of increasing amounts of Dz-155 (lane 3 -10pmole; lane 4 - 50pmoles; lane 5 100pmoles), no significant reduction in the amounts of input substrate RNA was observed. Lane 3 and 4 show no decrease but lane 5 shows 1.5 fold decrease when compared with lane 2 . On the other hand when the cleavage reaction was carried out in presence of increasing amounts of Dz-192 (lane 6 - 10 pmoles; lane $7-50$ pmoles and lane 8 100pmoles), a dose-dependent decrease in the input substrate RNA was observed with almost complete disappearance at 50 and 100pmoles of Dz-192. Lane6 shows 4 folds, lane 7 shows 5 folds and lane 8 shows 5.5 folds decrease when compared with input substrate RNA present in lane2 (Fig. 6A). We conclude that only the downstream Dz-192 was able to significantly enhance the Rz-170 mediated cleavage.

\section{Wild-Type \& Mutant Dz-192 Augment Rz-170 Mediated Cleavage}

The purpose of this experiment was to compare augmentation of the Rz-170 mediated cleavage between the wildtype and mutant-Dz-192 (disabled). As reported earlier, this mutant Dz [35] failed to cleave the target RNA completely and served as an important antisense control for the wildtype Dz. Exactly same amounts of wild-type (Dz-192) and mutant-Dz-192 were added to the cleavage reaction which contained equimolar amounts of Rz-170 and Substrate RNA. The results of this experiment are shown in Fig. (6B). Lane 1 is the $\mathrm{HBx}$ transcript and when cleavage reaction was carried out with Rz-170 alone (lane 2), two specific fragments were seen as described before. When the Rz-170 mediated cleavage reaction was carried out in the presence of increasing amounts of Dz-192 (lane 3 -1pmole; lane 4 -10pmoles and lane 5 -100pmoles), a dose-dependent decrease in the substrate RNA was observed along with multiple cleaved fragments. These fragments match the predicted pattern of cleavage described earlier (Fig. 5, steps C and D). The same experiment was carried out with increasing amounts of MutantDz-192 under identical conditions of cleavage (lane 6 1pmole; lane 7 -10pmoles and lane 8 -100pmoles). In this case also a dose-dependent decrease in the input substrate RNA was observed. In both cases, up to $8-10$ fold decrease in the input labeled substrate RNA was observed in the presence of highest amounts of Dz used (compare the extent of uncleaved input substrate RNA in lanes 1, 5 and 8). Since this mutant-Dz is catalytically inactive, no additional cleaved products were observed. The extent of reduction in the input RNA with either wild-type (WT) or mutant-Dz in corre-

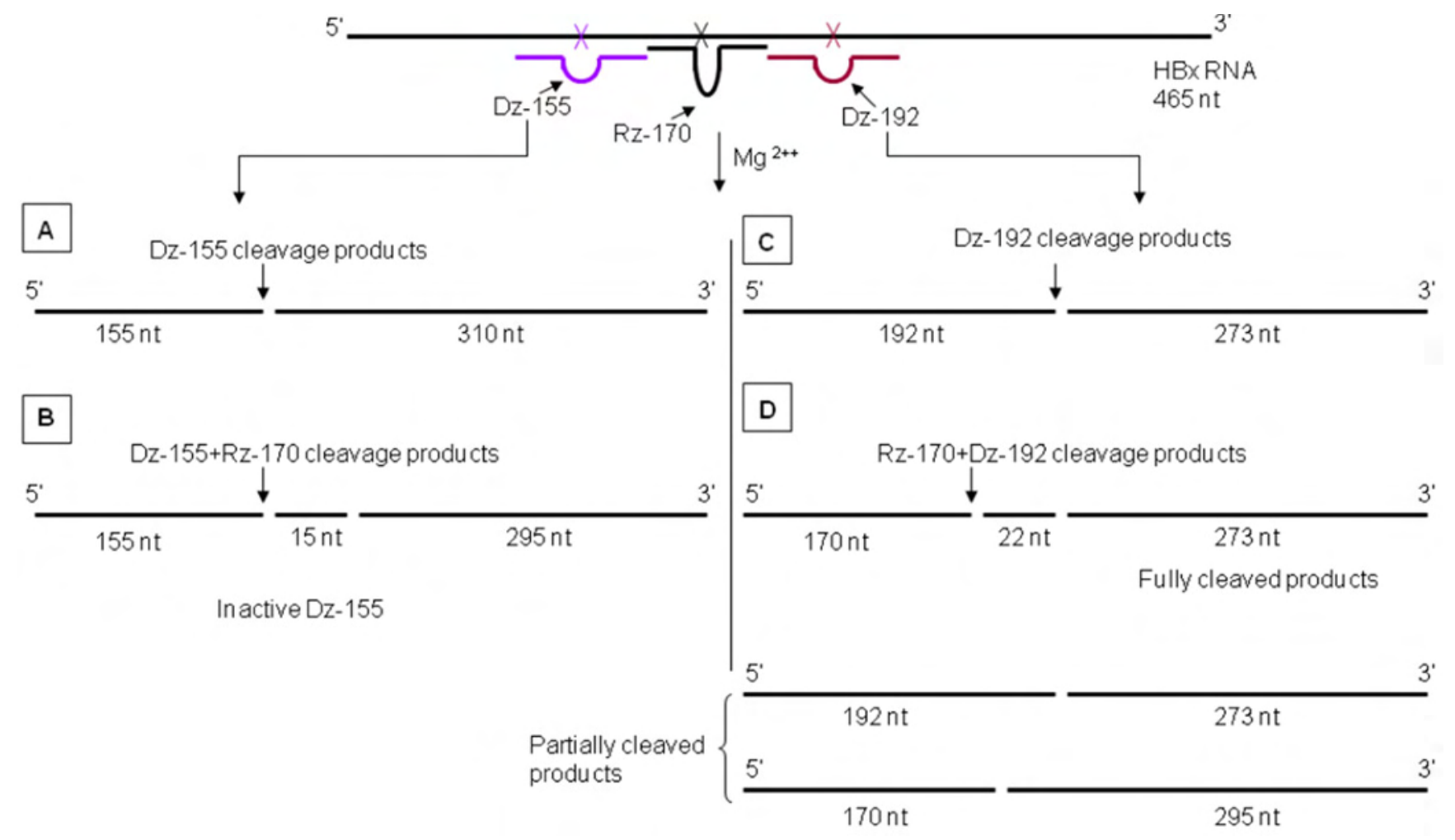

Fig. (5). Modulation of Rz-170 mediated cleavage products with Dzs.

Full-length HBx RNA was used as target RNA for cleavage with Rz-170 in the presence of upstream (Dz-155) and downstream Dz (Dz192). The expected cleavage pattern generated by cleavage with $\mathrm{Rz}, \mathrm{Dz}$ and $\mathrm{Rz}+\mathrm{Dz}$ is shown schematically in Fig. (6) (steps marked A to D). 
A.

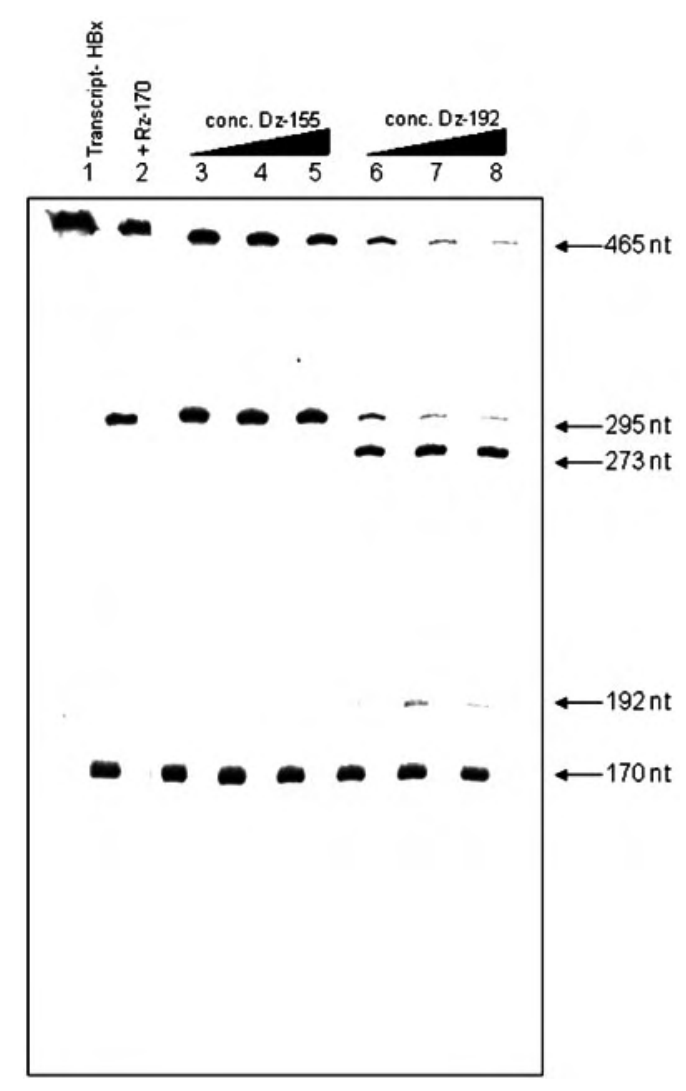

B.

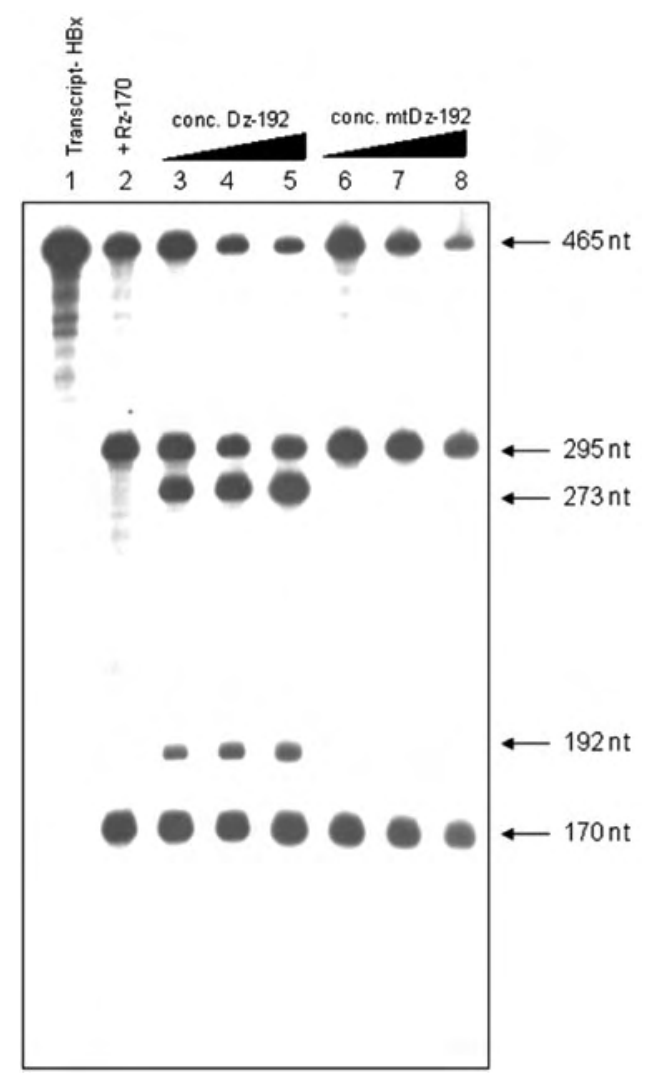

Fig. (6A). Downstream Dz (Dz-192) augments Rz-170 mediated cleavage very significantly.

Rz-170 mediated cleavage reaction was performed in the presence of Dz-155 and 192 in increasing concentrations. Lane 1 shows the HBx RNA (465nt). As expected, Rz-170 reaction generated two specific fragments (lane 2). When increasing concentration of Dz-155 (lane 3 10pmoles; lane $4-50$ pmoles; lane 5 -100pmoles) was used in the reaction, no significant enhancement of cleavage was observed (lanes 3 -5). On the contrary increasing amounts of Dz-192 (lane 6 -10pmoles; lane 7 -50pmoles and lane 8 -100pmoles) was very effective in cleaving the target RNA (as evident by the reduced amounts of input RNA remaining in lane 7 and 8).

Fig. (6B). Both wild-type and mutant Dz-192 show increased cleavage of the target RNA.

The purpose of this experiment was to compare the ability of catalytically active and inactive Dz-192 in their ability to augment Rz-170 cleavage of the target RNA (lane 1) under exactly identical conditions. Lane 2 depicts the extent of cleavage obtained with Rz-170 alone. When increasing amounts of Dz-192 (lane 3 -1pmole; lane 4 -10pmoles; lane 5 -100pmoles) was added to the reaction mixture, a dosedependent increase in the cleavage products was observed that matched the predicted pattern as shown in Fig. (5D). In the same manner, in presence of increasing amounts of mutant-Dz-192 (lane 6-1pmole; lane 7 -10pmoles; lane 8 -100pmoles), also resulted in dose-dependent cleavage of the substrate RNA.

sponding lanes were very similar. This indicated that the reduction in the input substrate RNA is mainly due to antisense effect and not because of catalytic activity. We conclude that both wild-type and mutant Dz-192 increased the Rz-170 mediated cleavage products to similar extent.

\section{HBx-Dz-237 Mediated Cleavage is Enhanced Moderately by Upstream but Powerfully by Downstream ODN}

All the earlier experiments described above were designed to increase the cleavage of Rz-170 and we next wanted to know if the earlier identified $\mathrm{Dz}$ against $\mathrm{HBx}$ RNA, Dz-237 [35] could also be modulated by using 20nt long upstream or downstream ODNs (Fig. 7A). The cleavage results of this experiment are shown in Fig. (7B). Lane 1 is the input substrate HBx RNA and lane 2 shows the Dz-237 mediated specific cleavage products ( 237 \& 228nt long RNA fragments). In the presence of increasing amounts of upstream ODN (ODN\#4) (lane 3 -10pmoles; lane 4 100pmoles; lane 5 -200 pmoles), only about 2 fold decrease in the amounts of uncleaved substrate RNA was observed with 200pmoles of ODN\# 4 (compare lane 4 with lane 2). When the same reaction was carried out in the presence of increasing amounts of downstream ODN (ODN\# 5) (lane 6 10 pmoles; lane 7 -100pmoles; and lane 8 -200pmoles), significant reduction in the substrate RNA was observed (> 20 fold reduction) with almost complete disappearance in the presence of 200pmoles of ODN\# 5 lane 8. We conclude that downstream ODN is significantly more effective in enhancing the Dz-237 mediated cleavage reaction. 
A.

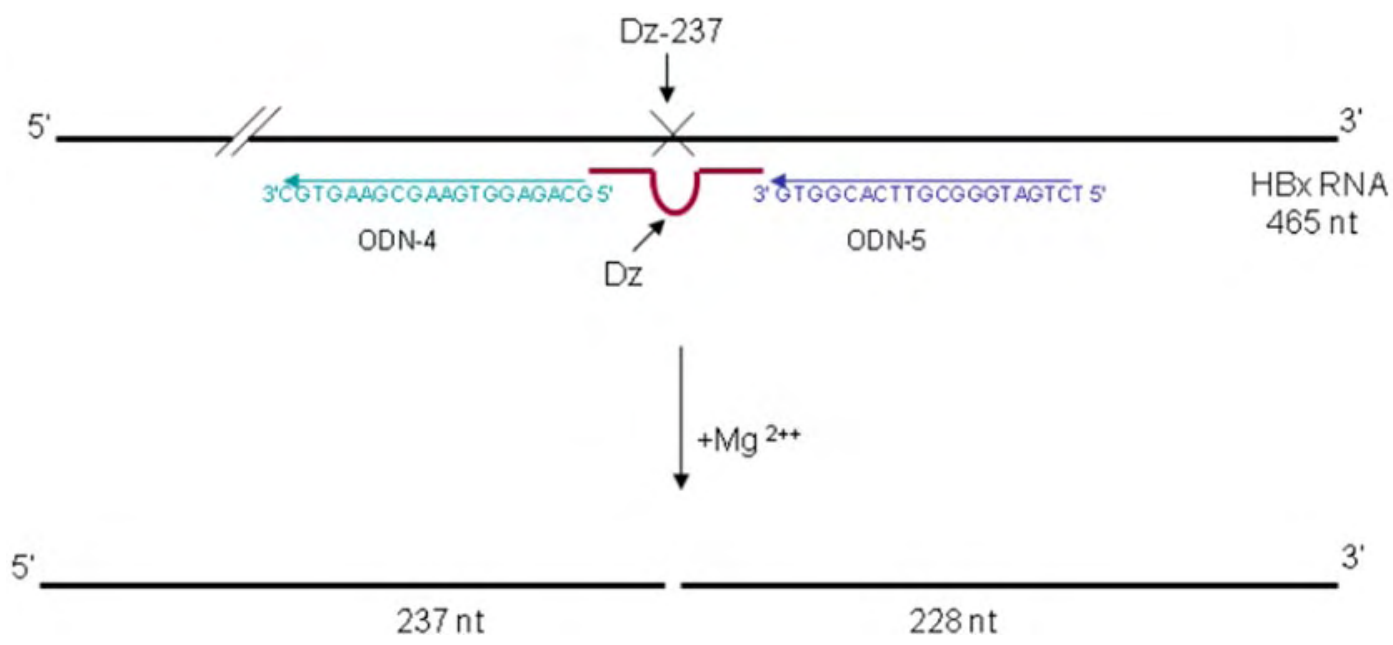

B.

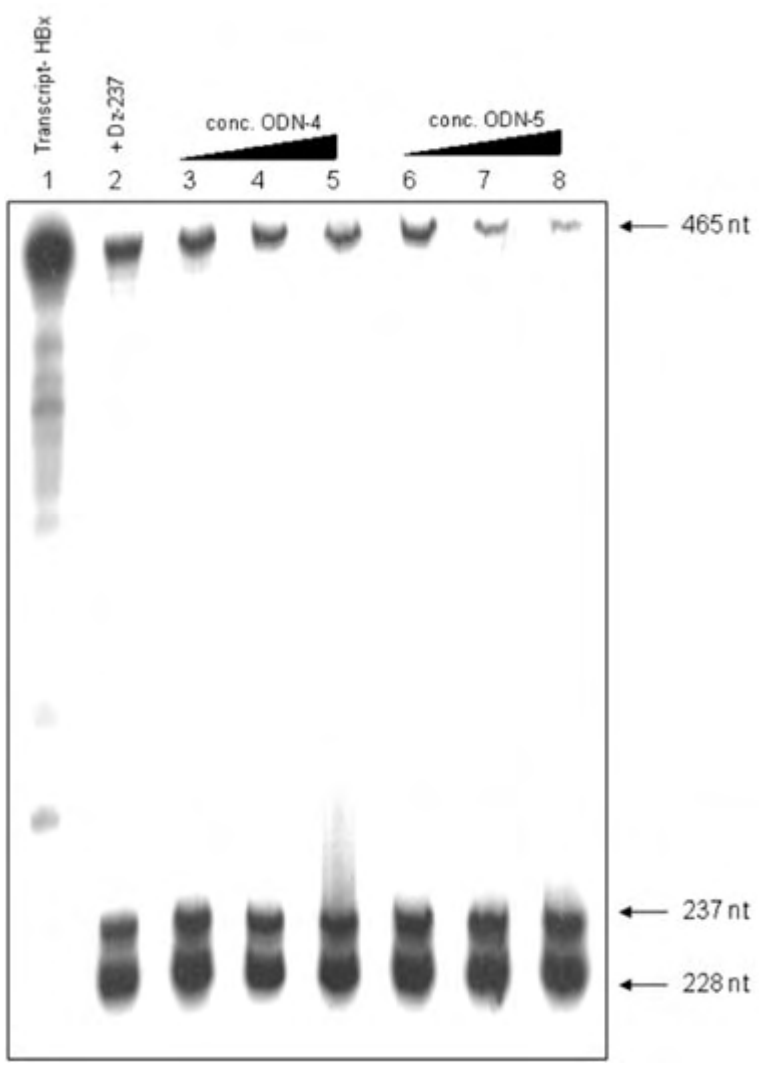

Fig. (7A). ODNs designed for augmenting Dz-237 mediated cleavage.

Dz-237 was used to cleave the target RNA in the presence of either ODN \#4 or 5. The sequences of the two ODNs flanking the hybridizing arms of the Dz-237 are also shown along with the expected cleaved fragments. Panel 7B: Lane 1 is the substrate RNA and lane 2 exhibits the cleavage products due to Dz-237 cleavage. In the presence of increasing concentrations of ODN\#4 (lane 3 - 10pmoles; lane 4 -100pmoles; lane 5 -200pmoles), a dose-dependent decrease in the input substrate RNA was observed. Similar observations were made with ODN\# 5 but it was more effective in decreasing the input substrate RNA when used in increasing amounts (lane $6-10$ pmoles; lane 7 -100pmoles and lane 8 - 200pmoles).

\section{Rz-170 + ODNs Treatment Results in Potent Intracellu- lar Reduction of Target RNA}

HepG2 cells were cotransfected with $1 \mu \mathrm{g}$ each of pSG5.HBx DNA and Rz-170 construct in one $\mathrm{ml}$ for 48 hours using $10 \mu \mathrm{l}$ of Lipofectin (Invitrogen). This dose was predetermined to give about $10 \%$ intracellular reduction in the levels of target RNA (Fig. 8, lane 4) when compared with cells transfected with pSG5.HBx DNA alone along with 
equal amounts of unrelated Rz (lane 3). Total RNA was isolated using TRIZOL and target RNA and control RNA were estimated simultaneously by RT-PCR technique. When $4 \mu \mathrm{g}$ of ODN \#2 was added to the Rz-170 construct + pSG5.HBx.DNA mixture (lane 5), approximately $70 \%$ reduction was observed (compare lane 5 with lane 3 ). When the same amount of ODN \#1 was used, about $20 \%$ reduction was observed (lane 6). When half the above amount of ODN

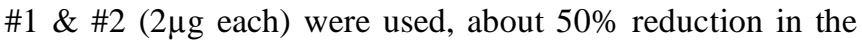
target RNA was observed (compare lane 7 with lane 3). RTPCR carried out with cells only (lane 2) and RT-PCR carried out in the absence of RT (data not shown), as expected, showed no HBx RNA specific amplification. The extent of house keeping gene (HuGAPDH) in all the corresponding lanes remained essentially unchanged. We conclude that Rz$170+$ downstream ODN combination was more effective in causing decrease in the levels of intracellular target RNA.

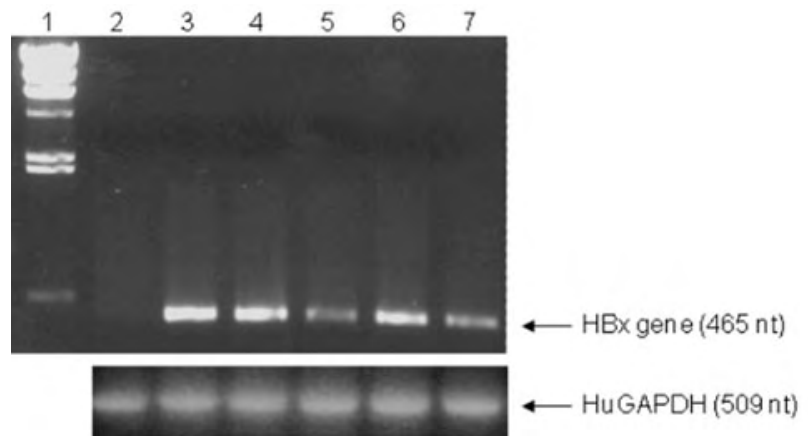

Fig. (8). Intracellular reduction of target RNA in the presence of $\mathrm{Rz}+$ ODNs.

Estimation of target RNA (HBx RNA) in HepG2 cells treated with various combinations of $\mathrm{Rz}$ and $\mathrm{Rz}+\mathrm{ODN} \# 1$ and \#2 by RT-PCR techniques. The levels of full-length $\mathrm{X}$ RNA and control RNA (HuGAPDH) were determined simultaneously by RT-PCR as described in materials and methods. Wells that received $1 \mu \mathrm{g}$ of pSG5.HBx DNA + equivalent amounts of unrelated ODN of similar length, showed a prominent $X$ gene-specific band. This amount was kept constant in all the wells. Cells were cotransfected with $1 \mu \mathrm{g}$ each of Rz-170 + pSG5.HBx DNA (lane 4); pSG5.HBx +1 $\mu \mathrm{g} \mathrm{Rz}+$ $4 \mu \mathrm{g}$ of ODN \#2 (lane 5); pSG5.HBx $+1 \mu \mathrm{g} \mathrm{Rz}+4 \mu \mathrm{g}$ of ODN \#1 (lane 6); pSG5.HBx $+1 \mu \mathrm{g} \mathrm{Rz}+2 \mu \mathrm{g}$ of ODN \#1 $+2 \mu \mathrm{g}$ of ODN \#2 (lane 7).

\section{Western Blot Analysis}

HepG2 cells cotransfected with Rz + ODNs were processed for total protein and subjected to western blot analysis as described before $[35,33]$ and the results are shown in Fig. (9). Lane 1 represents control cells only. A prominent immunoreactive $\mathrm{HBx}$ protein was observed (lane 2) when transfected with $1 \mu \mathrm{g}$ each of pSG5.HBx plasmid DNA. About 2-fold reduction in the HBx protein band was observed when cells were cotransfected with $1 \mu \mathrm{g}$ each of pSG5.HBx plasmid DNA and Rz-170 DNA (lane 3). When

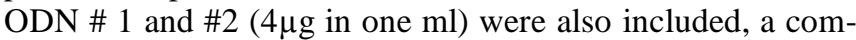
plete knock down of the HBx protein was observed (lanes 4 $\& 5$ ). This is not due to unequal amounts of cell lysates that were analyzed because equivalent amounts of cells containing same amounts of total protein were loaded (as determined by colorimetric reaction with BCA reagent from Pierce). We conclude that both the ODNs in combination with Rz-170 caused complete intracellular reduction in the levels of $\mathrm{X}$ protein.

\section{Potent Knock Down of HBx Mediated HIV-1 LTR Re- porter Gene Activation by Rz-170 + ODNs or 10-23 Dz}

HepG2 cells were cotransfected with pSG5.HBx + pLTR-B plasmid DNA (100 ng each in one $\mathrm{ml}$ for $1 \mathrm{X} 10^{6}$ cells) along with indicated amounts of ODNs or Dz and the reporter gene activity (luciferase) (mean +/- SD) obtained from three independent experiments are shown in Fig. (10). As expectd HBx encoding DNA showed significant LTRpromoter activity (compare lane 4 with lane 3 ). This activity diminished more than 2.4-fold when 100ng of Rz-170 was added (lane 5). Upon addition of ODN\#1, it dropped 8.4 fold (lane 6), with ODN\#2 about 5.8 fold (lane 7) and in combination of both about 8.4-fold (lane 8). Control cells and pSG5.HBx treated cells showed no significant reporter gene activity as expected (lanes $1 \& 2$ ). Thus, an additional $3-4$ fold decrease (when compared with Rz-170 alone) in X gene mediated reporter gene activity with ODN \#1 is observed. More than 17-fold reduction in reporter gene activity was observed with Dz-192 under identical conditions (lane 9) and about 12.6-fold reduction with the mutant version of Dz-192 (lane 10). We conclude that ODNs (\#1\&\#2), wild-type and mutant Dz-192 treated cells showed impressive functional inhibition of HBx protein. As expected Dz-192 (lane 11) and mutant Dz-192 (lane 12) showed significant reduction in reporter gene activity.

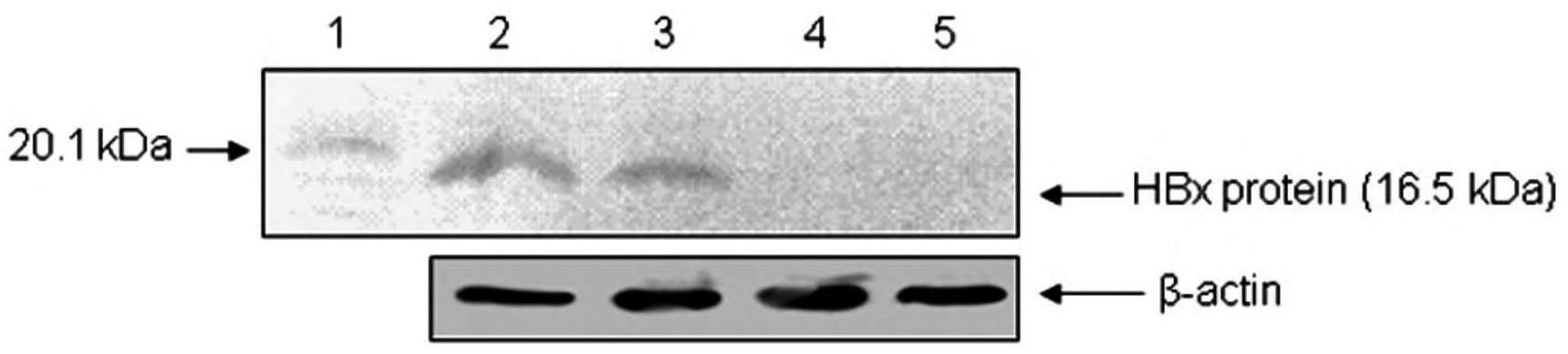

Fig. (9). Intracellular reduction in the levels of $X$ protein by western-blot analysis.

HepG2 cells were transfected with pSG5.HBx plasmid alone (lane 2); cotransfected with pSG5HBx + Rz-170 construct (lane 3); pSG5HBx + Rz-170 construct + ODN \#1 (lane 4); pSG5HBx + Rz-170 construct + ODN \#2 (lane 5) with indicated amounts (see result section). The total amount of DNA used in each well was kept constant by adding unrelated or unrelated ODNs of similar length. Cell lysates were prepared from equivalent amount of cells and subjected to western blot analysis as described before. 


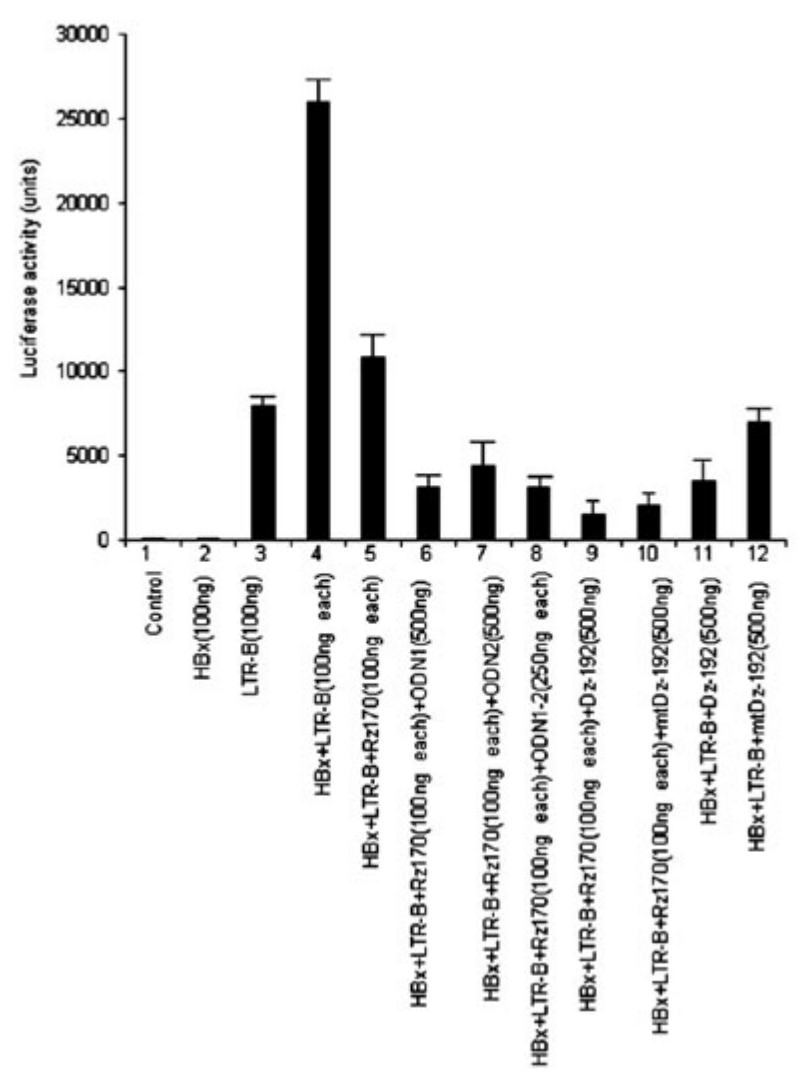

Fig. (10). Inhibition of $X$ protein mediated HIV-1 LTR reporter gene activation with $\mathrm{Rz}+\mathrm{ODNs}$.

293 or HepG2 cells were grown to $80 \%$ confluency in 24 well plate and then transfected with various combinations of plasmid DNAs as indicated + ODNs or 10-23 Dzs as shown in the bottom of the figure using $2.5 \mu$ of Lipofectin (Invitrogen) per well. 24 hours post transfection equivalent amounts of cells were subjected to lysis using $1 \mathrm{x}$ reporter lysis buffer (Promega) and the extent of luciferase activity (light units on Y axis) was determined as described earlier (Goila \& Banerjea, 2001).

\section{DISCUSSION}

Efficient cleavage of a target RNA by Rz or Dz is important for several reasons including biochemical and gene therapy approaches. Although there are several potential Dz or $\mathrm{Rz}$ target sites in a target RNA, most of them are cleaved poorly or not at all when tested on longer and physiologically relevant RNAs. We report that ODNs that were targeted to hybridize directly adjacent to the Rz or Dz target sites were able to significantly augment $\mathrm{Rz}$ or $\mathrm{Dz}$ mediated cleavage activity in dose-dependent manner and that the downstream $\mathrm{Dz}$ or ODNs usually worked better than the upstream Dz. In both the cases ( $\mathrm{Rz}+\mathrm{ODNs}$ or $\mathrm{Dz}+\mathrm{ODNs})$ almost complete cleavage of the target RNA was achieved under defined set of conditions. Showing close to $100 \%$ in vitro cleavage of the target RNA with Rz or Dz in the presence of specific ODNs or Dz on one hand and potent intracellular reduction in a mammalian liver-specific cell line on the other are the major findings of the present work. It must be emphasized that all the previous studies that employed either $\mathrm{Rz}$ or $\mathrm{Dz}$ alone, complete in vitro disappearance of the input substrate RNA was never achieved under standard conditions of cleavage. This data assumes more significance because ODNs and Dzs are currently being exploited for therapeutic purposes $[6,11]$. The most likely explanation for enhanced cleavage by $\mathrm{Rz}$ or Dz with specific ODNs is their ability to melt the secondary structure near the $\mathrm{Rz}$ and $\mathrm{Dz}$ target sites which facilitated efficient hybridization between Rz-170 or Dz-237 with HBx RNA. This ODN mediated enhancement of cleavage was not observed with unrelated ODN of similar length (data not shown) or with ODN that hybridized 33nt upstream of the target RNA. This is most probably because long RNAs are known to exist in separate stem-loop structures or independent domains. Results with upstream and downstream (wild-type and mutant Dz-192) are somewhat surprising but not totally unexpected. The upstream Dz-155 not only failed to increase the cleavage activity of Rz-170 but also was catalytically inactive as no cleavage products were seen. It is likely that Dz-155 failed to hybridize with the target RNA because of the secondary structure constraints. In this connection it is important to note that the ability of ODNs to successfully hybridize to the target RNA (as measured by RNAse $\mathrm{H}$ based assay) is essential but not sufficient for affording catalytic activity to Dz [37]. It is noteworthy that the hybridization arms of the Dz or Rz span only 14 or $16 \mathrm{nt}$ (with one gap in the design) in the target RNA whereas the ODNs are uninterrupted 20nt long, which is likely to melt secondary structures better. The advantage of using downstream $\mathrm{Dz}$ would obviously be its ability to cleave the target RNA in a catalytic manner. A comparative Rz-170 mediated study between wild-type and mutant Dz192 , suggests that the enhancement of Rz-170 cleavage is largely due to the antisense effects because the amounts of 
input substrate RNA after various treatments remained the same. Similar intracellular level of reduction of target RNA was also observed with Dz-237 + ODN\#5 (data not shown).

Although very few studies on enhanced cleavage by nucleic acid approaches have earlier been reported, we report, for the first time, that a combination of Rz-170 and specific ODNs or 10-23 Dz lead to not only complete cleavage of the target RNA but can also cause very substantial intracellular reduction of $\mathrm{X}$ RNA. The ability of ODNs to augment in vitro cleavage by Rz-170 correlated with their ability to interfere with the intracellular expression of the target gene.

In summary, we show that both $\mathrm{Rz}$ and $\mathrm{Dz}$ mediated cleavage of the full-length HBx RNA can be powerfully augmented by specific ODNs and 10-23 Dzs. They not only enhanced the $\mathrm{Rz} \& \mathrm{Dz}$ mediated in vitro cleavage products, but also reduced very significantly the intracellular target gene expression in a mammalian liver specific cell line HepG2. The intracellular effectiveness of these ODNs or Dzs could be substantially improved by using chemically modified (stabilized) nucleotide [37]. These observations are of immediate therapeutic importance and should be applicable to down regulating other target genes also.

\section{ACKNOWLEDGEMENTS}

This work was supported by the funds from Department of Biotechnology, Government of India to ACB and National Institute of Immunology, New Delhi, India.

\section{ABBREVIATIONS}

$\begin{array}{lll}\text { ODNs } & =\text { Oligodeoxynucleotides } \\ \mathrm{Dz} & =\text { DNA-enzyme } \\ \mathrm{Rz} & =\text { Ribozymes } \\ \mathrm{RT} & =\text { Reverse transcriptase } \\ \mathrm{HBV} & =\text { Hepatitis B virus } \\ \mathrm{LTR} & =\text { Long terminal repeat }\end{array}$

\section{REFERENCES}

[1] Cech TR. The chemistry of self-splicing RNA and RNA enzymes.Science 1987; 236: 1532-39.

[2] Haseloff J, Gerlach W. Simple RNA enzymes with new and highly specific endoribonuclease activities. Nature 1998; 334: 585-91.

[3] Uhlenbeck OC. A small catalytic oligoribonucleotide. Nature 1987; 328: 596-600.

[4] Rossi JJ. Controlled, targeted, intracellular expression of ribozymes: Progress and problems. Trends Biotechnol 1995; 13: 301-06.

[5] Kurreck J. Antisense technologies: Improvement through novel chemical modifications. Eur J Biochem 2003; 270: 1628-44.

[6] Schubert S, Kurreck J. Oligonucleotide-based antiviral strategies. Handb Exp Pharmacol 2006; 173: 261-87.

[7] Banerjea A, Mingg-Ji Li, Remling L, Rossi JJ, Akkina R. Lentiviral transduction of Tar Decoy and CCR5 ribozyme into CD34+ progenitor cells and derivation of HIV-1 resistant $\mathrm{T}$ cells and macrophages. AIDS Res Ther 2004a; $17^{\text {th }}$ December, online.

[8] Akkina R, Banerjea A, Bai J, Anderson J, Li M J, Rossi JJ. siRNAs, ribozymes, and RNA decoys in modeling stem cell based gene therapy for HIV/AIDS. Anticancer Res 2003; 23 : 1997-05.

[9] Santoro SW, Joyce GF. A general purpose RNA-cleaving DNA enzyme. Proc Natl Acad Sci 1997; 94: 4262-66.

[10] Silverman SK. Survey and Summary: In vitro selection, characterization, and application of deoxyribozymes that cleave RNA. Nucleic Acids Res 2005; 33: 6151-63.
[11] Banerjea AC, Chakraborti S, Unwalla H, et al. Potential therapeutic application of DNA enzymes and siRNAs against viral and cellular genes. In: synthetic nucleic acids as inhibitors of gene expression: mechanisms, applications, and therapeutic implications. Khachigian LM, ed. (CRC Press, Florida, USA) 2004; 115-34.

[12] Joyce GF. Directed evolution of nucleic acid enzymes. Ann Rev Biochem 2004; 73: 791-36.

[13] Chakraborti S, Banerjea AC. Inhibition of HIV-1 gene expression by novel DNA enzymes targeted to cleave HIV-1 TAR RNA:Potential effectiveness against all HIV-1 isolates. Mol Ther 2003; 7: 817-26.

[14] Dash BC, Banerjea AC. Sequence-specific cleavage activities of DNA-enzymes targeted against HIV-1 Gag and Nef regions. Oligonucleotides 2004; 14: 41-47.

[15] Ackermann JM, Kanugula S, Pegg AE. DNAzyme-mediated silencing of ornithine decarboxylase. Biochemistry 2005; 44: 214352.

[16] Goila R, Banerjea AC. Sequence-specific cleavage of HIV-1 coreceptor-CCR5 gene by a hammerhead ribozyme and a DNAenzyme: Inhibition of the coreceptor function by DNA-enzyme. FEBS Letters 1998; 436: 233-38.

[17] Kurreck J, Bieber B, Jahnel R, Erdmann VA. Comparative study of DNA enzymes and ribozymes against the same full-length messenger RNA of the vanilloid receptor subtype 1. J Biol Chem 2002; 277: 7099-07.

[18] Cairns MJ, Hopkins TM, Witherington C, Wang L, Sun LQ. Target site selection for an RNA-cleaving catalytic DNA. Nat Biotechnol 1999; 17: 480-86.

[19] Wadhwa R, Ando H, Kawasaki H, Taira K, Kaul SC. Targeting mortalin using conventional and RNA-helicase-coupled hammerhead ribozymes. EMBO Rep 2003; 4: 595-01.

[20] Grunweller A, Wyszko E, Bieber B, Jahnel R, Erdmann VA, Kurreck J. Comparison of different antisense strategies in mammalian cells using locked nucleic acids, 2'-O-methyl RNA, phosphorothioates and small interfering RNA. Nucleic Acids Res 2003; 31: 3185-93.

[21] Schubert S, Furste JP, Werk D, et al. Gaining target access for deoxyribozymes. J Mol Biol 2004; 339: 355-63.

[22] Wang DY, Sen D. A novel mode of regulation of an RNA-cleaving DNAzyme by effectors that bind to both enzyme and substrate. J Mol Biol 2001; 310: 723-34.

[23] Wang DY, Beatrice HY, Lai BH, Feldman AR, Sen D. A general approach for the use of oligonucleotide effectors to regulate catalysis of RNA-cleaving ribozymes and DNAzymes. Nucleic Acids Res 2002; 30: 1735-42.

[24] Goodchild J. Enhancement of ribozyme catalytic activity by a contiguous oligonucleotide (facilitator) and by 2'-O-methylation Nucleic Acids Res 1992; 20: 4607-12.

[25] Horn S, Schwenzer B. Oligonucleotide facilitators enhance the catalytic activity of RNA-cleaving DNA enzymes. Antisense $\mathrm{Nu}-$ cleic Acid Drug Dev 1999; 9: 465-72.

[26] Jankowsky EB, Schwenzer B. Oligonucleotide facilitators may inhibit or activate a hammerhead ribozyme. Nucleic Acids Res 1996; 24: 423-29.

[27] Sood V, Gupta N, Bano AS, Banerjea AC. DNA-ezyme mediated cleavage of human immunodeficiency virus type 1 Gag RNA is significantly augmented by antisense-DNA molecules targeted to hybridize close to the cleavage site. Oligonucleotides 2007; 17 : 113-21.

[28] Jankowsky EB, Schwenzer B. Oligonucleotide facilitators enable a hammerhead ribozyme to cleave long RNA substrates with multiple-turnover activity. Eur J Biochem 1998; 254: 129-34.

[29] Robinson WS. Molecular events in pathogenesis of hepadnaviruses associated hepatocellular carcinoma. Annu Rev Med 1994; 45: 297-23.

[30] Gomezganzalo M, Carretero M, Rullas J, et al. The hepatitis B virus $\mathrm{X}$ protein induces HIV-1 replication and transcription in synergy with T-cell activating signals. Functional roles of NfkappaB/NF-AT and SP1-binding sites in the HIV-1 long terminal repeat promoter. J Biol Chem 2001; 276: 35435-43.

[31] Kumar V, Jayasuryan N, Kumar R. A truncated mutant (residues $58-140$ ) of the hepatitis B virus X protein retains transactivation function. Proc Natl Acad Sci 1996; 93: 5647-52.

[32] Banerjea AC, Joklik WK. Reovirus protein sigma 1 translated in vitro as well as truncated derivatives of it that lack up to two-thirds of its C-terminal portion, exists as two major tetrameric molecular 
species that differ in electrophoretic mobility. Virology 1990; 179: $460-62$.

[33] Goila R, Banerjea AC. Sequence specific cleavage of hepatitis X RNA in cis and trans by novel monotarget and multitarget hammerhead motif-containing ribozymes. Oligonucleotides 2004; 14 : 49-62.

[34] Shahi S, Shanmugasundaram GK, Banerjea AC. Ribozymes that cleave reovirus genome segments $\mathrm{S} 1$ also protect cells from pathogenesis caused by reovirus infection. Proc Natl Acad Sci USA 2001; 98: 4101-06.
[35] Goila R, Banerjea AC. Inhibition of hepatitis B virus X gene expression by novel DNA enzymes. Biochem J 2001; 353: 701-08.

[36] Paik S, Banerjea A, Harmison GG, Chen C-J, Schubert M. Defective HIV-1 provirus encoding a multitarget ribozyme inhibits accumulation of spliced and unspliced HIV-1 mRNAs, reduces infectivity of viral progeny and protects the cells from pathogenesis. Hum Gene Ther 1997; 8: 1115-24.

[37] Kurreck J, Wyszko E, Gillen C, Erdman VA. Design of antisense oligonucleotides stabilized by locked nucleic acids. Nucleic Acids Res 2002; 30: 1911-18. 